\title{
Editorial: Blood Groups in Companion Animals
}

\author{
Elizabeth B. Davidow ${ }^{1 *}$, Eva Spada ${ }^{2}$ and Daniela Proverbio ${ }^{2}$ \\ ${ }^{1}$ Veterinary Clinical Sciences, College of Veterinary Medicine, Washington State University, Pullman, WA, United States, \\ ${ }^{2}$ Veterinary Transfusion Research Laboratory (REVLab), Department of Veterinary Medicine (DIMEVET), University of Milan, \\ Lodi, Italy
}

Keywords: gel typing, feline AB blood type, DEA blood group, Dal blood type, Kai 1, card typing

\section{Editorial on the Research Topic}

Blood Groups in Companion Animals

Blood transfusions can be life-saving but carry the risk of acute and delayed immunologic reactions. Reaction risk can be mitigated with a better understanding of blood groups. Blood groups are determined by the expression, or lack of expression, of red blood cell (RBC) surface antigens. Their importance in transfusion compatibility was first identified in people in the early 1900s and ABO blood-typing started in the 1920s. Canine blood groups were also discovered during this time, but were not further defined until the 1950s (1). The DEA (dog erythrocyte antigen) system in dogs and the $\mathrm{AB}$ system in cats are the most recognized blood groups. Other groups, including $\mathrm{Dal}$ and $\mathrm{Kai}$ in dogs and $\mathrm{MiK}$ in cats, have now been identified. To continue to improve the safety of transfusions, we need to understand the geographic and breed distribution of blood groups, improve blood-type identification in dogs and cats, and further understand the genetics and antigenicity of blood groups.

This collection advances our knowledge of blood groups in dogs and cats. Ebelt et al., in "Survey

OPEN ACCESS

Edited and reviewed by: Tracy Stokol,

Cornell University, United States

*Correspondence:

Elizabeth B. Davidow

elizabeth.davidow@wsu.edu

Specialty section: This article was submitted to Veterinary Experimental and

Diagnostic Pathology,

a section of the journal

Frontiers in Veterinary Science

Received: 11 October 2021 Accepted: 05 November 2021 Published: 30 November 2021

Citation:

Davidow EB, Spada E and Proverbio D (2021) Editorial: Blood Groups in Companion Animals.

Front. Vet. Sci. 8:792720.

doi: 10.3389/fvets.2021.792720 of Blood Groups DEA 1, DEA 4, DEA 5, Dal, and Kai 1/Kai 2 in Different Canine Breeds from a Diagnostic Laboratory in Germany," present typing results in 206 dogs. Incidence data for DEA antigens and for the Dal and Kai groups, using a gel column technique (ID-card, DiaMed, Cressier, Switzerland), are included. This study also describes newly available methods in-house typing with agglutination cards using polyclonal antisera identifying DEA 4 and 5 (RapidVet-H, DMS, Flemington, NJ, USA).

As in previously reports, $100 \%$ of dogs were positive for DEA 4 . The study also identified that 96.6\% of dogs were Kai $1+$ and Kai 2-. Five Lhasa Apsos and one Maltese were Kai 1-/Kai 2+ and one Maltese was Kai 1-/Kai 2-. While Dal- was originally described in Dalmatians, and only sporadically in other breeds such as the Cane Corso (2), this study identified additional breeds without Dal and demonstrated the need for more widespread typing. The gel test used to blood type in this study is not commercially available.

DEA 5 was typed by both the card and gel tests for 158 of the 206 samples. Results matched in $97 \%$ with five discordant results. Four of the five discordant results were negative on the card test, but positive on the gel test. The cards were easy to use but weak agglutination reactions on initial testing were difficult to interpret, leading the authors to double the dilution of blood. The increased dilution strengthened some of the weak reactions and led to a change in the manufacturer's instructions to help account for the weak agglutination. This in-house test for DEA 5 improves our ability to further study the clinical relevance of this blood type.

McDermott et al. in "The Prevalence of Blood Groups in Domestic Cats in the Saskatoon and Calgary Areas of Saskatchewan and Alberta, Canada” describe typing results in 400 cats from two regions in Canada. Previous large-scale studies of blood type incidence in North American cats are 
almost three decades old. Due to increased movement of people and animals, continued geographic incidence blood-type surveys are warranted. In this study, cats were typed using the Rapid $\mathrm{H}$ gel test (DMS Laboratories, Flemington, NJ, USA). Type A was identified in $384(96 \%)$, type B in $16(4 \%)$, and no type AB cats were identified. These were healthy domestic cats and did not include pedigree cats. Because the incidence of type $A B$ is low, it is hard to interpret whether the lack of any $A B$ cats is due to geographic distribution or related to typing methodology.

The accuracy of different typing methodologies in cats was explored in Spada et al. in "Comparison of Conventional Tube and Gel-Based Agglutination Tests for AB System BloodTyping in Cats." This study compared the gold standard tube agglutination with a gel column system (ID-card, DiaMed, Cressier, Switzerland). Gel typing is used extensively in human medicine. In this study, mixed field reactions were occasionally seen with cells remaining at the top of the gel and a larger population settling at the bottom. Previously, Seth et al. noted a mixed field pattern in gel typing for some cats with FeLV (3). Thus, retroviral seropositivity was explored as a possible reason for the mixed field pattern. However, this relationship was not significant $(p=0.17)$. When mixed field gels were viewed as negative, there was $98.6 \%$ concordance with the tube methodology. The two discrepancies were one cat typed as B by the gel but $\mathrm{AB}$ by the tube and one typed as $\mathrm{AB}$ by the gel but type $B$ by the tube. While the gel test has important time and reproducibility advantages, cats who type as $\mathrm{B}$ or $\mathrm{AB}$ by this method should ideally be confirmed by another method.

Uno et al. in "Phenotypic and Genetic Characterization for Incompatible Cross-Match Cases in the Feline AB Blood Group System" report two cats blood-typed as B on the card test (RapidVet-H, Kyoritsu Seiyaku Corporation, Tokyo, Japan) but found to be type $\mathrm{AB}$ on tube agglutination. Both cats had incompatible major crossmatches to type B RBCs. Genetic testing was pursued to investigate the discrepancy. The enzyme, cytidine monophosphate- $\mathrm{N}$-acetylneuraminic acid hydroxylase

\section{REFERENCES}

1. Wardrop KJ. New red blood cell antigens in dogs and cats - a welcome discovery. J Vet Intern Med. (2007) 21:2056. doi: 10.1111/j.1939-1676.2007.tb02949.x

2. Proverbio D, Lubas G, Spada E, Medina Valentin AA, Viñals Florez LM, del Rosario Perlado Chamizo M, et al. Prevalence of Dal blood type and dog erythrocyte antigens (DEA) 1, 4, and 7 in canine blood donors in Italy and Spain. BMC Vet Res. (2020) 16:1-5. doi: 10.1186/s12917-020-02351-1

3. Seth M, Jackson KV, Giger U. Comparison of five blood-typing methods for the feline AB blood group system. Am J Vet Res. (2011) 72:2039. doi: 10.2460/ajvr.72.2.203

4. Davidow EB, Blois SL, Goy-Thollot I, Harris L, Humm K, Musulin $\mathrm{S}$, et al. Association of veterinary hematology and transfusion medicine (AVHTM) transfusion reaction small animal consensus statement (TRACS) part 2: prevention and monitoring. J Vet Emerg Crit Care. (2021) 31:16788. doi: $10.1111 /$ vec. 13045

5. Cooling L. Blood groups in infection and host susceptibility. Clin Microbiol Infect. (2015) 28:801-70. doi: 10.1128/CMR.00109-14

6. Anstee DJ. The relationship between blood groups and disease. Blood. (2010) 115:4635-43. doi: 10.1182/blood-2010-01-261859

7. Yazer MH, Judd WJ, Davenport RD, Dake LR, Lomas-Francis C, Hue-Roye $\mathrm{K}$, et al. Case report and literature review: transient Inab phenotype and an
(CMAH) changes $\mathrm{N}$-acetylneuraminic acid (Neu5AC), expressed by type B RBCs, to N-glycolylneuramini acid (Neu5Gc), which is expressed by type A RBCs. The two cats had different variants in the CMAH gene that likely led to the low level of $\mathrm{A}$ antigen. The lack of identification of $\mathrm{A}$ antigen by the card in some $\mathrm{AB}$ cats was described in a previous paper (3). This study provides genetic information about why A antigen might be hard to identify in $\mathrm{AB}$ cats. Crossmatching does identify incompatibilities in cats with discordant typing results. These results support the Association of Veterinary Hematology and Transfusion Medicine's (AVHTM) Transfusion Reaction Small Animal Consensus (TRACS) statement recommending that all cats be blood-typed and crossmatched prior to transfusion (4).

Further research on blood groups is needed. This collection provides evidence that the results of blood-typing and crossmatching are method-dependent and this should be acknowledged when interpreting and comparing studies. While we are gaining understanding of the role of blood groups in veterinary transfusion medicine, there is a paucity of information about the additional roles of RBC antigens. In humans, certain types of blood groups may impact infectious disease risk and disease expression $(5,6)$. In addition, some gastrointestinal diseases and cancer can alter blood antigen expression $(7,8)$. The interaction of disease and blood groups is an interesting area that warrants additional exploration in veterinary medicine.

\section{AUTHOR CONTRIBUTIONS}

All authors listed have made a substantial, direct, and intellectual contribution to the work and approved it for publication.

\section{ACKNOWLEDGMENTS}

Thank you to all the authors that contributed to this Research Topic.

agglutinating anti-IFC in a patient with a gastrointestinal problem. Transfusion. (2006) 46:1537-42. doi: 10.1111/j.1537-2995.2006.00933.x

8. Nambiar RK, Narayanan G, Prakash NP, Vijayalakshmi K. Blood group change in acute myeloid leukemia. Baylor Univ Med Cent Proc. (2017) 30:745. doi: 10.1080/08998280.2017.11929536

Conflict of Interest: The authors declare that the research was conducted in the absence of any commercial or financial relationships that could be construed as a potential conflict of interest.

Publisher's Note: All claims expressed in this article are solely those of the authors and do not necessarily represent those of their affiliated organizations, or those of the publisher, the editors and the reviewers. Any product that may be evaluated in this article, or claim that may be made by its manufacturer, is not guaranteed or endorsed by the publisher.

Copyright (c) 2021 Davidow, Spada and Proverbio. This is an open-access article distributed under the terms of the Creative Commons Attribution License (CC BY). The use, distribution or reproduction in other forums is permitted, provided the original author(s) and the copyright owner(s) are credited and that the original publication in this journal is cited, in accordance with accepted academic practice. No use, distribution or reproduction is permitted which does not comply with these terms. 